\title{
Mejoras de la regulación de frecuencia utilizando el aumento de inercia de microrredes interconectadas
}

\section{Regulation frequency improvements using the inertia increase of networked microgrids}

\author{
Vladimir Toro-Tovar ${ }^{1}$, Sergio Rivera ${ }^{2}$, Eduardo Mojica-Nava $^{3}$ \\ ${ }^{1}$ Programa de investigacion sobre adquisicion y analisis de señales PAAS-UN, Universidad Nacional de Colombia, Colombia. \\ Email: bwtorot@unal.edu.co \\ ${ }^{2}$ Grupo de Investigación de Compatibilidad Electromagnética EMC-UN, Universidad Nacional de Colombia, Colombia. \\ Email: sriverar@unal.edu.co \\ ${ }^{3}$ Programa de investigacion sobre adquisicion y analisis de señales PAAS-UN, Universidad Nacional de Colombia, Colombia. \\ Email: eamojican@unal.edu.co
}

RECIBIDO: Agosto 19, 2016. ACEPTADO: Marzo 20, 2017. Versión FInAL: Mayo 30, 2017.

\begin{abstract}
RESUMEN
El control por pendiente garantiza la regulación de frecuencia y el reparto de potencia activa entre los inversores de una microrred aislada, sin embargo, introduce desviaciones respecto a la frecuencia de referencia. Cuando un generador adicional basado en inversores se conecta a una microrred, se reduce la desviación en frecuencia y se aumenta la capacidad de potencia de todo el sistema; de igual modo, la interconexión entre microrredes en modo isla tiene el mismo efecto. El control por pendiente ha mostrado ser efectivo en microrredes basadas en inversores, sin embargo, cuando uno o más generadores sincrónicos se conectan directamente a la microrred, cambian las condiciones de dicho control. Para analizar que ocurre cuando se interconectan microrredes en modo isla se analizan los casos cuando ambas microrredes constan únicamente de inversores, y cuando una microrred o ambas tienen al menos un generador sincrónico. De esta manera, los resultados de simulación presentados demuestran las mejoras de la regulación de frecuencia utilizando el aumento de inercia de microrredes interconectadas.
\end{abstract}

PALABRAS ClAVE: Microrred, Control por pendiente, Frecuencia, Interconexión, Generador sincrónico, Regulación.

\begin{abstract}
Droop control guarantees frequency regulation and power sharing among the inverters in an isdated microgrid, however, frequency deviations are introduced with respect to reference values. When an aditional distributed generator based on inverters is connected to the microgrid, such deviations are reduced and the power capacity of the system is improved; same way happens when microgrids are interconnected. Droop control has shown to be effective in inverter based microgrids, however, when one or more synchronous generators are directly connected to the microgrid the conditions of the controller change. To analaize what happen when islanded microgrids are interconected different cases are considered: first when both microgrid are based only in inverters, and other case in which one microgrid has at least one synchronous generator. In this way, the simulation results show the regulation frequency improvements using the inertia increase of networked microgrids.
\end{abstract}

KEYWORDS: Microgrid, Droop control, Frequency, Networked, Synchronous generator, Regulation.

Este artículo puede compartirse bajo la licencia CC BY-ND 4.0 y debe referenciarse usando el siguiente formato: V. Toro, S. Rivera, E. MojicaNava, "Mejoras de la regulación de frecuencia utilizando el aumento de inercia de microrredes interconectadas", UIS Ingenierías, vol. 16, no. 2, pp. 35-42, Julio-Diciembre 2017. Doi: https://doi.org/10.18273/revuin.v16n2-2017003 


\section{INTRODUCCIÓN}

El incremento en el uso de fuentes alternativas de energía, como la eólica y la solar, ha aumentado la necesidad de modificar los sistemas de potencia tradicionales. En el futuro los sistemas de potencia irán cambiando hacia las denominadas redes inteligentes (Smart Grids). El concepto de redes inteligentes es muy amplio, incluyendo aspectos de generación distribuida, gestión de la demanda, medición inteligente, entre otros [1]. Una unidad básica para el desarrollo de las redes inteligentes es la microrred (Microgrid), está puede definirse como una agrupación de cargas y generadores distribuidos que operan como una unidad controlable que provee potencia a su área ya sea aislada o conectada a la red de potencia convencional [2]. Según [3] las microrredes pueden simplificar la puesta en funcionamiento de muchas características de las redes inteligentes. Por ejemplo, el sistema de distribución puede ser dividido en zonas dentro de las cuales varias microrredes se interconectan, disminuyendo la necesidad de un sistema de comunicaciones más complejo. Los sistemas actuales de potencia están implementando proyectos de microrredes en sus redes, de esta manera, en el mediano y largo plazo habrá múltiples áreas interconectadas a través de estas microrredes con elementos propios de las redes inteligentes. Las empresas de energía deben tener herramientas de coordinación y control que puedan manejar sus actividades cooperativamente ante los diferentes disturbios que se presenten en el sistema de potencia.

De esta manera, las microrredes son la manera práctica en que se ha incrementado la penetración de energías no convencionales, con 5 tipos de elementos básicos que las conforman: las fuentes de energía tradicionales, las fuentes de energías renovables, las cargas controlables, las cargas no controlables y los sistemas de almacenamiento de energía [4], [5].

El control por pendiente es una técnica ampliamente utilizada en microrredes, éste reduce las altas corrientes circulantes que pueden aparecer al conectar inversores en paralelo, a la vez que garantiza el reparto de potencia de acuerdo con la capacidad de cada inversor. En una microrred aislada, los inversores ajustan su frecuencia de acuerdo a sus rangos de máxima potencia activa desviándose de la frecuencia nominal. Así, un sistema cuya demanda de potencia está cerca de su rango máximo, está más desviado de su frecuencia nominal. Si se interconectan dos o más microrredes, no sólo se ajusta su frecuencia acercándola más a su valor nominal, sino que mejora su regulación de potencia ante la conexión de grandes cargas. Este efecto se produce por las inercias propias de los diferentes elementos de los sistemas. Una microrred basada únicamente en inversores tiene una inercia muy baja, por otra parte, otras microrredes incluyen generadores sincrónicos, cuya inercia es mucho más alta y que puede ser determinante en la respuesta del sistema. La presente investigación aporta conocimiento en el modelado a través de computador (Computer Based Modeling) de este tipo de sistemas, que se puede categorizar como un sistema complejo en la teoría de sistemas de control.

Este documento está organizado de la siguiente forma: la sección 2 introduce aspectos generales para el control de microrredes A.C. y el control por pendiente. La sección 3 establece las generalidades para la conexión de generadores sincrónicos a microrredes. La sección 4 establece tres escenarios de conexión de microrredes dependiendo si estas tienen generadores sincrónicos. La sección 4 muestra algunos resultados de simulación en SimPower de Matlab. Finalmente, en la sección 5 se presentan las conclusiones.

\section{CONTROL DE LA GENERACIÓN EN MICRORREDES}

En una microrred A.C. se conectan en paralelo varios inversores de voltaje, debido a que es la tecnología más utilizada con fuentes renovables. Una microrred conectada al sistema de distribución trabaja como una fuente de corriente, en donde las referencias de voltaje y frecuencia son dadas por el sistema de potencia. Una microrred en modo isla, funciona como una fuente de voltaje, generando sus propias referencias de voltaje y frecuencia. Para garantizar la estabilidad y el reparto proporcional de la potencia entre los inversores de una microrred, la técnica conocida como control por pendiente es ampliamente utilizada. Este tipo de control se basa en un desacople entre la potencia activa y reactiva que suple el inversor. La potencia activa es controlada mediante variaciones en la frecuencia y la potencia reactiva es controlada mediante variaciones en el voltaje.

De acuerdo con la teoría de sistemas de potencia, se pueden hacer varias generalizaciones de un inversor conectado a un bus de voltaje por medio de una línea de transmisión [6].

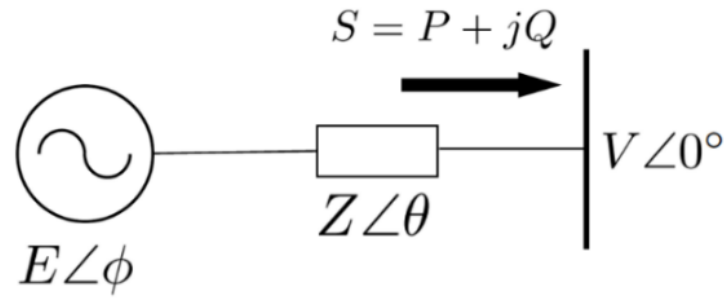

Figura 1. Conexión entre un inversor y el bus de voltaje.

La Figura 1 muestra la conexión de una fuente de voltaje a un bus de conexión común a través de una línea de 
transmisión, donde $\phi$ es el ángulo de fase entre el voltaje de salida del inversor y el voltaje del bus común. $E$ es la amplitud del voltaje de salida del inversor y $V$ es la amplitud del voltaje en el bus común.

Para líneas de transmisión altamente inductivas $Z \angle \theta=X \angle 90^{\circ}$ se cumple:

$$
\begin{aligned}
& P=\frac{E V}{X} \sin \phi \\
& Q=\frac{E V \cos \phi-V^{2}}{X}
\end{aligned}
$$

Donde $P$ y $Q$ son los valores de potencia medidos y $X$ el valor de la reactancia de la línea de transmisión.

De (1) y (2) se pueden obtener las ecuaciones de control por pendiente para una microrred con línea de transmisión predominantemente inductiva [7].

$$
\begin{aligned}
& \omega=\omega_{r e f}-m_{p} P \\
& E=E_{r e f}-m_{q} Q
\end{aligned}
$$

Donde $\omega_{\text {ref }}$ es la referencia de frecuencia angular, $E_{\text {ref }}$ es el valor de referencia de voltaje, $m_{p}$ y $m_{q}$ son los coeficientes de control por pendiente para potencia activa y potencia reactiva, respectivamente.

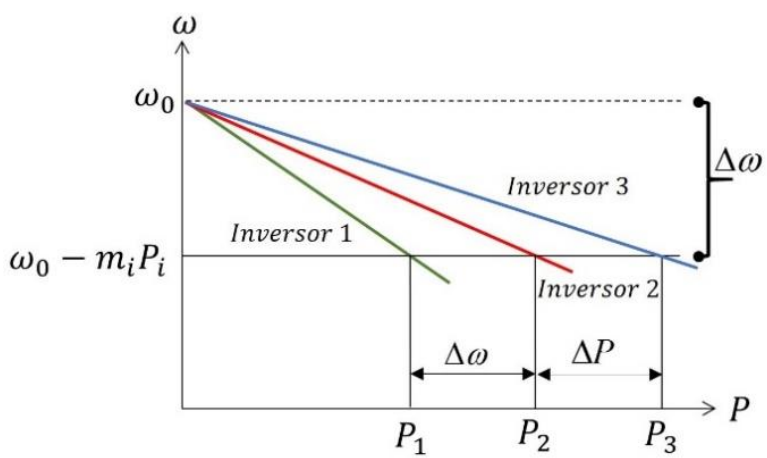

Figura 2. Control por pendiente $P$ - $f$.

La Figura 2 presenta el control de pendiente $P$ - $f$ (o $P$ - $\omega$ ) para tres inversores. Al cambiar la demanda de potencia activa, cada inversor ajusta su frecuencia de acuerdo a su coeficiente de control por pendiente, en estado estacionario todos los inversores alcanzarán el mismo valor de frecuencia. Los valores de $\Delta P$ son diferentes y dependen de los rangos máximos de potencia activa de cada inversor.
La asunción de las relaciones $P-f$ y $Q-V$ y el desacople entre las potencias activa y reactiva sólo es válida para redes de alto voltaje [8], en redes de bajo voltaje, la relación puede ser inversa, es decir, $P-V$ y $Q-f$, como se muestra en la Figura 3:

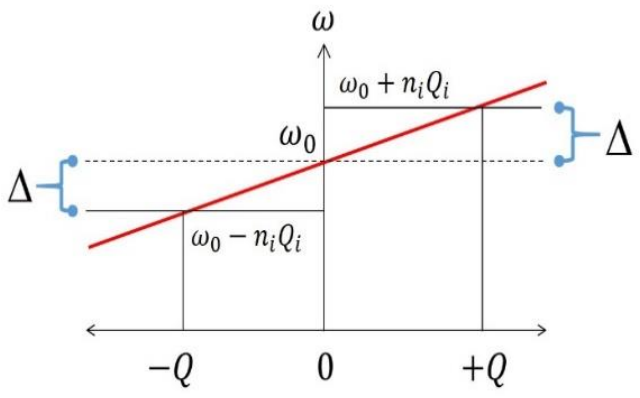

Figura 3. Control por pendiente $Q-f$.

Para garantizar un desacople de potencia y una relación $P-f$ y $Q-V$ adecuados, se utiliza una impedancia virtual en cada inversor, que consiste en un lazo de re-alimentación de corriente multiplicado por el valor de la impedancia virtual [8], [9], [10].

$$
v_{r e f}=v_{0}^{*}-Z_{v}(s) i_{0}
$$

El voltaje de referencia para cada inversor, incluido el efecto de la impedancia virtual, aparece en (4), [11]. Donde $v_{\text {ref }}$ es el valor de referencia de voltaje para los lazos de control de voltaje y corriente, $v_{0}^{*}$ es el voltaje de salida sin carga, $Z_{V}(s)$ es la impedancia virtual de salida y $i_{0}$ es la corriente de salida del inversor. El diagrama de conexión para la impedancia virtual se muestra en la Figura 4.

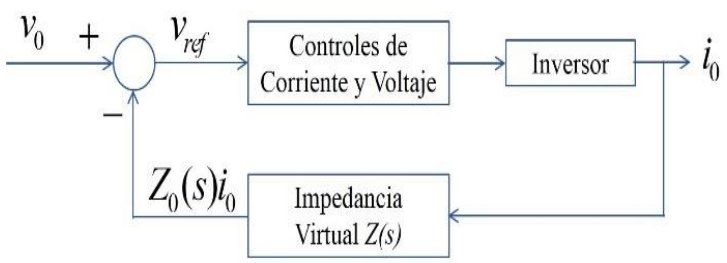

Figura 4. Diagrama de bloques para la técnica de impedancia virtual [12].

El control por pendiente requiere calcular la potencia activa y reactiva sobre un ciclo y pasarlas por un filtro pasa-bajos (Ver Figura 5) cuyo ancho de banda es menor que el ancho de banda del inversor en ciclo cerrado [13].

Un esquema general para el control por pendiente $P-f$ se muestra a continuación: 


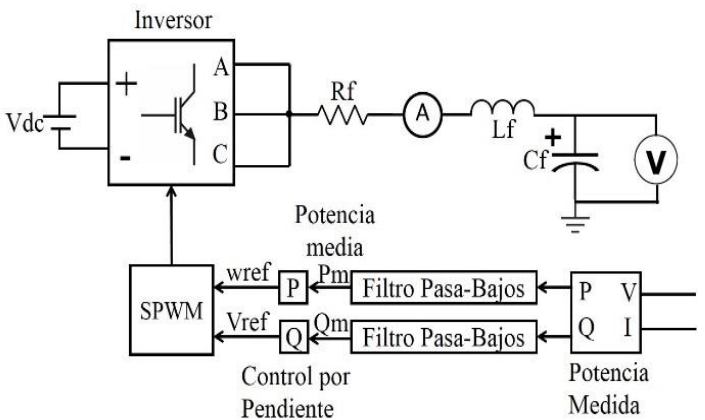

Figura 5. Esquema general del control por pendiente.

Los coeficientes del control por pendiente y los filtros determinan la dinámica y estabilidad del inversor [14]. Si el valor de $m_{p}$ se incrementa, se logra el reparto de potencia (power sharing), pero la regulación se degrada [13]. La acción del control por pendiente está limitada por la máxima desviación en frecuencia y voltaje.

Los coeficientes del control por pendiente $m_{p}$ y $m_{q}$ pueden ser escogidos según ([14]).

$$
m_{p}=\frac{\Delta \omega}{P_{\max }} \quad m_{q}=\frac{\Delta E}{Q_{\max }}
$$

El reparto de potencia es una propiedad deseable en un sistema de potencia con varios inversores, esto garantiza que cada inversor entregará una cantidad de potencia de acuerdo con sus rangos. Para asegurar esta propiedad, se debe cumplir la condición dada en ([15]).

$$
m_{1} P_{1}=m_{2} P_{2}=\cdots=m_{n} P_{n}
$$

Una de las desventajas del control por pendiente es la desviación introducida en voltaje y frecuencia de sus valores nominales. Este método funciona bien para cargas lineales, pero no es el más adecuado cuando las cargas son no-lineales [13].

Dado que todos los inversores deben llegar al mismo valor de frecuencia en estado estacionario. Se puede llegar a la siguiente igualdad que cumple con la condición dada en ([15])

$$
\frac{P_{1}}{P_{\max , 1}}=\frac{P_{2}}{P_{\max , 2}}=\cdots=\frac{P_{n}}{P_{\max , n}}
$$

La condición dada en (6) se mantiene para $n$ inversores.

\section{MICRORREDES CON GENERADORES SINCRÓNICOS}

Una microrred puede contener únicamente inversores o puede incluir generadores sincrónicos; en el segundo caso, la dinámica de la red varía considerablemente debido a la alta inercia de las máquinas sincrónicas comparada con la rápida respuesta de los inversores.

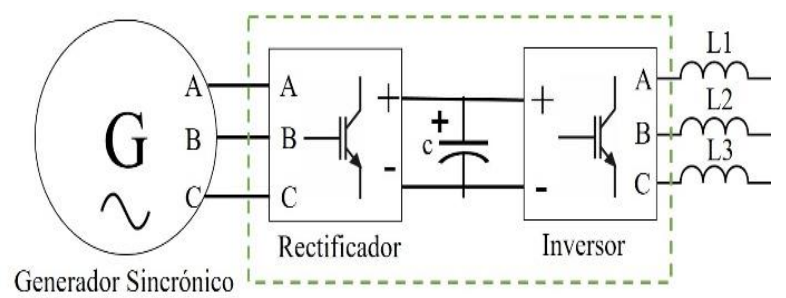

Figura 6. Generador sincrónico conectado a través de un conversor back to back.

En la mayoría de los casos, un generador sincrónico (SG) se conecta al bus común de una microrred $\mathrm{AC}$ a través de una interface de conversores denominada conversor back to back por su nombre en inglés (Ver Figura 6). Una primera etapa rectifica los voltajes de salida del SG, ésta etapa se conecta con un inversor por medio de un condensador en paralelo que garantiza un voltaje DC estable. La etapa inversora genera las señales trifásicas a la frecuencia y amplitud deseadas. La principal ventaja de este tipo de conexión es la generación de un conjunto de voltajes trifásicos estables aislados de las perturbaciones a que pueda estar sometido el generador. Una de las principales desventajas de esta conexión son las pérdidas producidas en cada una de las etapas, reduciendo la eficiencia del conjunto [15].

Un generador sincrónico puede conectarse directamente a una microrred, por ejemplo, un generador que funciona a base de diésel. Dado que el control por pendiente $P / f-$ $Q / V$, está basado en el funcionamiento de una máquina sincrónica, es natural utilizar esta propiedad para garantizar el reparto de potencia entre un SG y los generadores distribuidos de la microrred. La poca inercia de un inversor permite cambiar rápidamente la frecuencia y magnitud del voltaje en comparación con un SG, en el cual estos valores son determinados por la velocidad del rotor [15]. La dinámica de un SG es determinada por (7)

$M_{i} \dot{\theta}_{i}+D_{i} \dot{\theta}_{i}=P_{i}^{*}-P_{e, i}(\theta)$,

donde $M_{i}$ es la inercia, $D_{i}$ es el coeficiente de amortiguación, $P_{i}^{*}$ es la inyección de potencia de potencia mecánica cuyo valor es positivo para el caso de un generador, y $P_{e, i}(\theta)$ es la potencia eléctrica medida en el $i$-ésimo SG [16], [17].

\section{INTERCONEXIÓN DE MICRORREDES}

El modelado y simulación de los sistemas de potencia tradicionales requiere que cada elemento tenga una 
representación precisa y que la simulación sea el resultado de la solución de una serie de ecuaciones algebraicas y diferenciales que son bien conocidas [5]. Con la penetración de energías renovables en el sistema de potencia se deben reformular los modelos y por ende la ecuaciones que gobiernan el comportamiento con este tipo de energías en el sistema. Estos nuevos modelos y ecuaciones se deben centrar en la variabilidad en el tiempo de este tipo de energías.

Una microrred diseñada para funcionar como una unidad independiente, capaz de garantizar el suministro de potencia a las cargas aún cuando está aislada del sistema de distribución, puede conectarse con otras microrredes.

La conexión entre microrredes ayuda a mejorar el desempeño de cada una reduciéndo el estrés que puedan sufrir los inversores. Al disminuir la potencia activa demandada a cada inversor, se reduce el valor del término $m_{p} P$ en la ecuación del control por pendiente, mejorando la regulación en frecuencia y disminuyendo las oscilaciones generadas al conectar una carga grande al sistema.

Se pueden vislumbran tres casos diferentes al interconectar microrredes: 1. Dos microrredes basadas únicamente en inversores. 2. Una microrred basada en inversores y otra que tiene generadores sincrónicos. 3 . Dos microrredes, cada una con generadores sincrónicos.

\subsection{Conexión entre microrredes formadas únicamente por inversores}

La baja inercia de los inversores permite una variación rápida de la frecuencia ante cambios de carga. Permitiendo una mejor respuesta ante cargas variables, pero incrementando la posibilidad de comportamientos oscilatorios en frecuencia.

La Figura 7 muestra dos microrredes basadas en inversores que se interconectan a través de una línea de transmisión, cada microrred puede tener un rango de potencia máxima diferente. Para este caso se utiliza, generalmente, un segundo lazo de control que reestablesca los valores de referencia de voltaje y frecuencia antes de la inteconexión.

\subsection{Conexión de una microrred formada únicamente por inversores $y$ otra con al menos un generador sincrónico}

En una microrred con un generador sincrónico, la alta inercia de éste determina la respuesta del sistema ante cambios abruptos de carga.
La Figura 8 presenta la conexión entre una microrred basada en inversores y otra que tiene al menos un

generador sincrónico. En este caso, la microrred basada en inversores toma como referencia los valores de la microrred con el generador sincrónico.

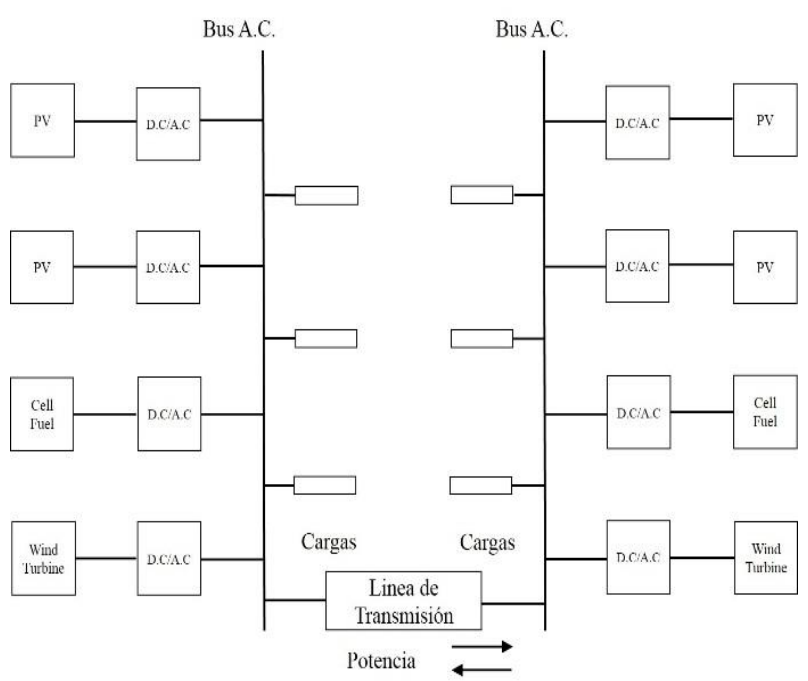

Figura 7. Esquema general de conexión entre microrredes basadas en inversores.

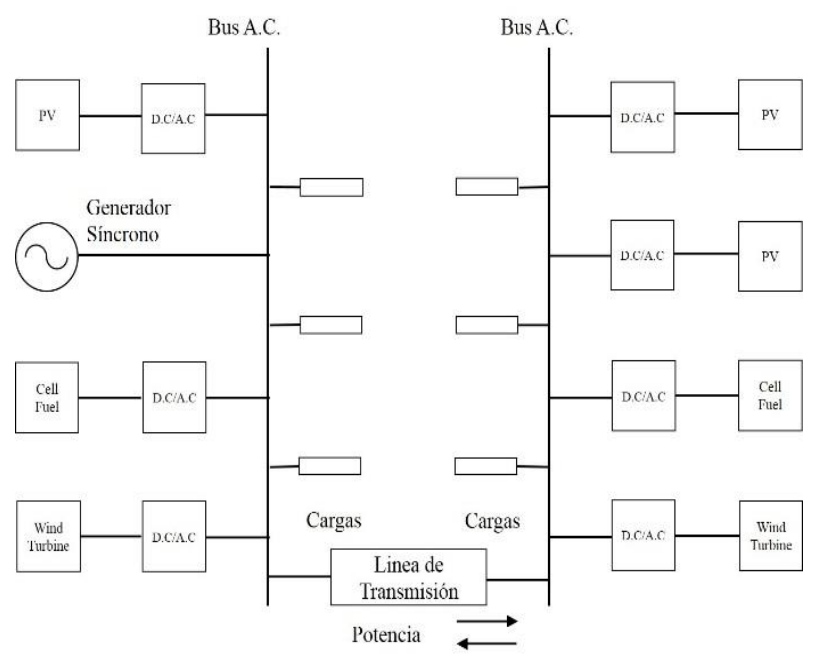

Figura 8. Esquema general de conexión entre dos microrredes: una con un generador sincrónico y otra que consta solo de inversores.

La Figura 8 presenta la conexión entre una microrred basada en inversores y otra que tiene al menos un generador sincrónico. En este caso, la microrred basada en inversores toma como referencia los valores de la microrred con el generador sincrónico. 
4.3 Conexión entre dos microrredes cada una con al menos un generador sincrónico

Al conectar dos microrredes que contienen generadores sincrónicos (Ver Figura 9), la frecuencia está determina por el generador con mayor inercia. En este caso, la conexión es similar a la de varios generadores sincrónicos.

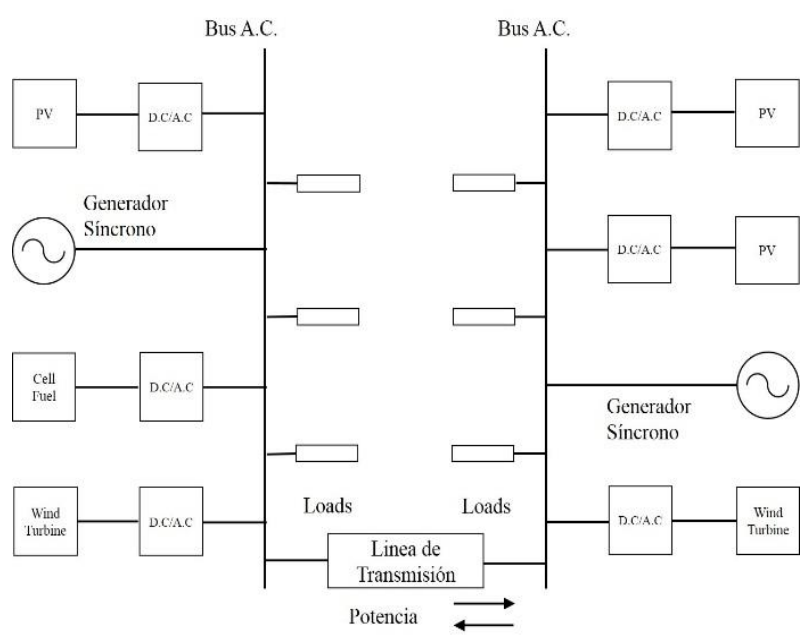

Figura 9. Esquema general de conexión entre microrredes cada una con al menos un generador sincrónico.

\section{Resultados}

Los escenarios simulados en PowerSim de Matlab ${ }^{\circledR}$ se basan en bus común de $400 \mathrm{Vrms}$, cada inversor utiliza el mismo filtro de potencia activa para el control primario, se garantiza que están en fase y que la magnitud de voltage es aproximadamente igual al momento de la conexión.

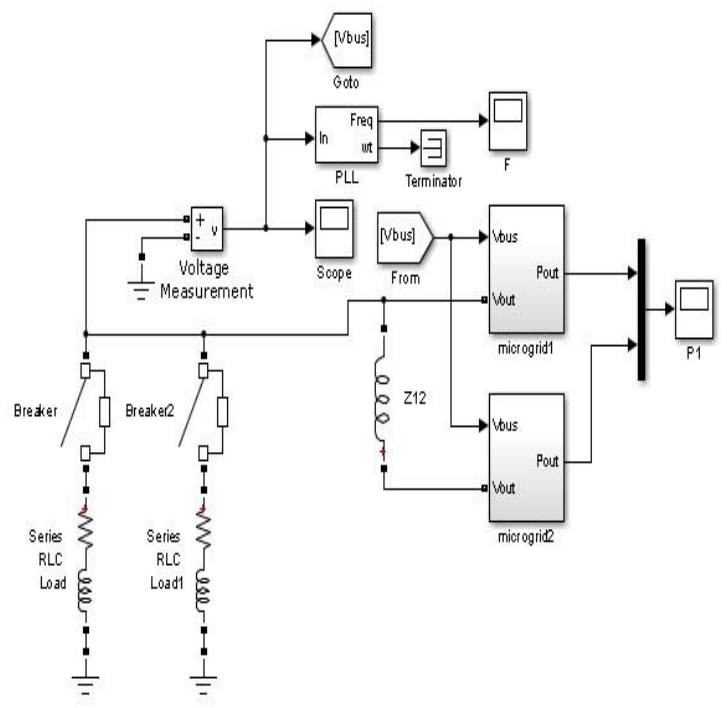

Figura 10. Diagrama de simulación para una microrred.
La Figura 10 muestra el esquema de conexión para dos microrredes a través de la línea de transmisión $Z_{12}$, las dos cargas se conectan al bus de la microrred 1. La Figura 11 muestra la forma en que se conectan tres inversores dentro de una microrred particular; para los casos dos y tres uno de los inversores es reemplazado por el SG..

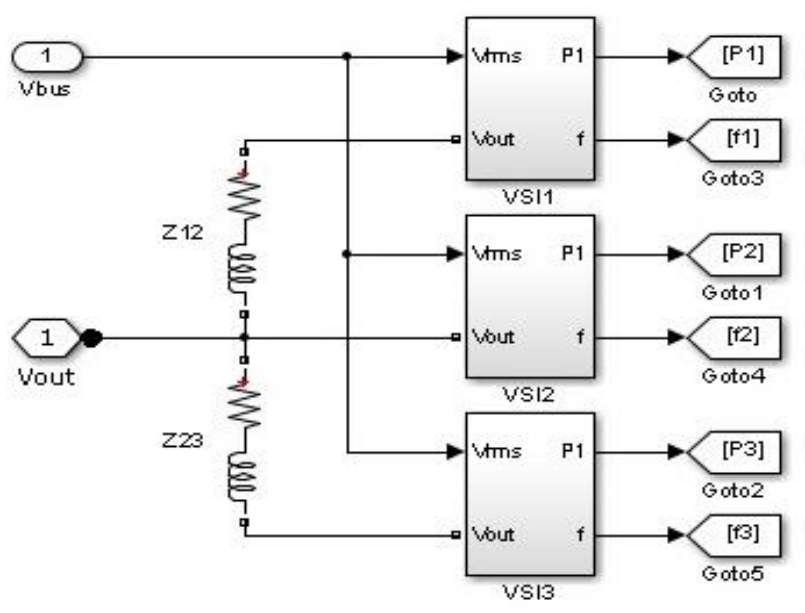

Figura 11. Diagrama de simulación para una microrred.

La Tabla 1 presenta los valores utilizados en una microrred basada en inversores.

Tabla1. Parámetros de una microrred.

\begin{tabular}{|c|c|c|c|}
\hline Parámetro & $\begin{array}{c}\text { Inversor } \\
1\end{array}$ & $\begin{array}{c}\text { Inversor } \\
2\end{array}$ & $\begin{array}{c}\text { Inversor } \\
3\end{array}$ \\
\hline $\begin{array}{l}\text { Frecuencia de } \\
\text { corte }(\mathrm{Hz})\end{array}$ & 4 & 4 & 4 \\
\hline $\begin{array}{l}\text { Potencia Activa } \\
\text { Nominal }(\mathrm{kW})\end{array}$ & 20 & 25 & 30 \\
\hline $\begin{array}{l}\text { Impedancia de } \\
\text { Línea }\end{array}$ & \multicolumn{3}{|c|}{$\begin{array}{l}Z_{12}=0.2 \Omega+j 0.37 \\
Z_{23}=0.12 \Omega+j 0.45\end{array}$} \\
\hline
\end{tabular}

Fuente: Elaboración propia.

Para el primer caso de simulación se interconectan dos microrredes basadas en inversores, ambas microrredes se asumen idénticas.

La Figura 12 muestra la variación de la frecuencia en el sistema para el primer caso. Inicialmente las microrredes están desconectadas y sin carga. Se conecta una carga de $50 \mathrm{~kW}$ a la microrred 1 en $t=2 s$, observándose una caída en la frecuencia respecto al valor nominal. En $t=4 \mathrm{~s}$, se conectan las dos microrredes mejorando la desviación de frecuencia. 


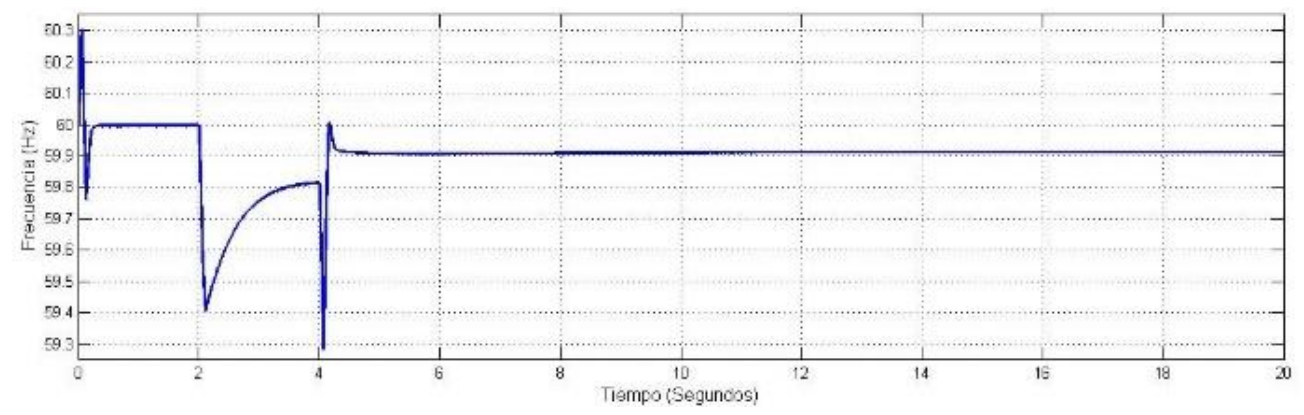

Figura 12. Variación de frecuencia para dos microrredes basadas en inversores.

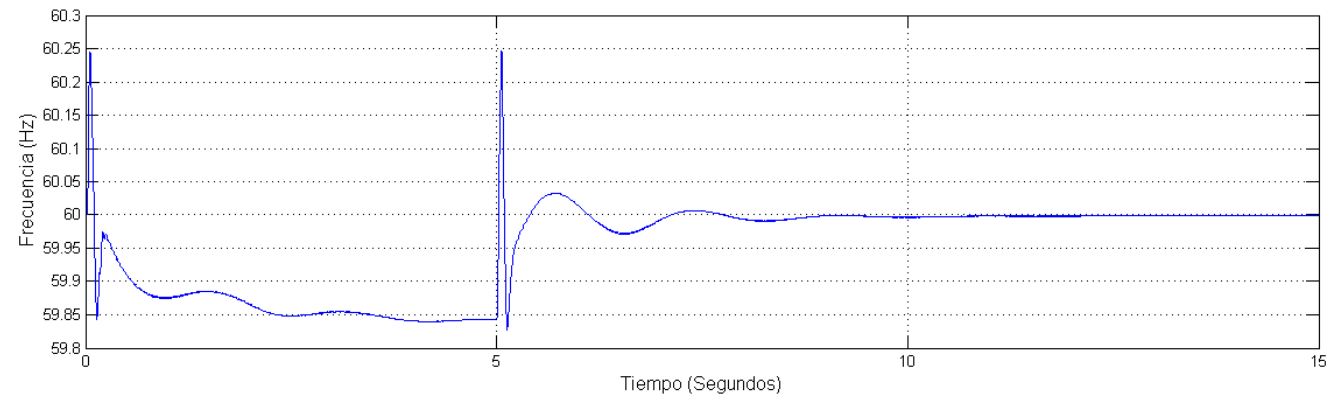

Figura 13. Variación de frecuencia para dos microrredes, una de las cuales tiene al menos un generador sincrónico.

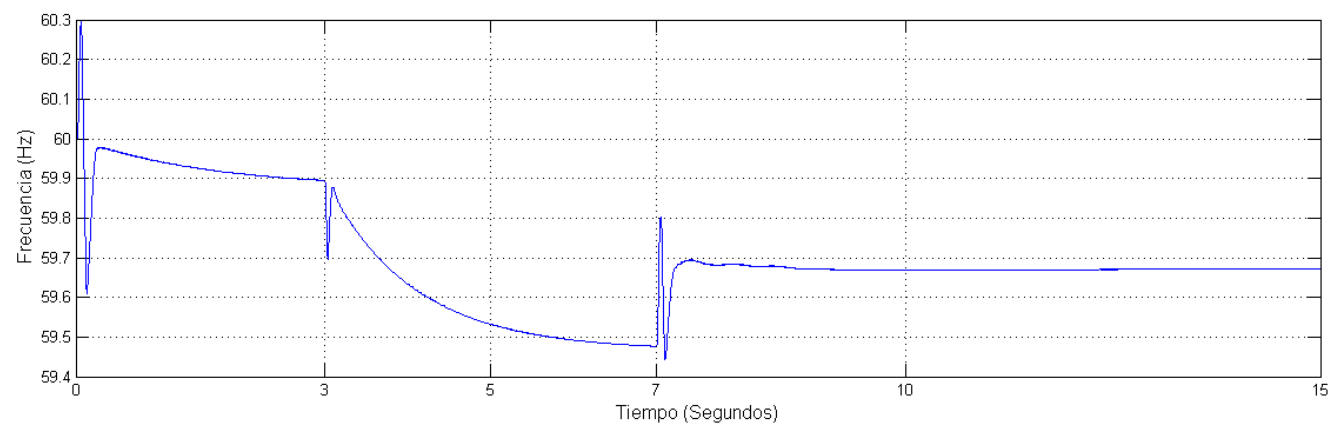

Figura 14. Variación de frecuencia entre microrredes cada una de los cuales tiene un generador sincrónico.

En el segundo caso, un invesor (el inversor de $20 \mathrm{~kW}$ ) es reemplazado por un generador sincrónico 460 Vrms 1-1, $10.2 \mathrm{kVA}, 1800 \mathrm{rpm}$, en una de las microrredes.

La Figura 13 muestra la variación de frecuencia para dos microrredes una de las cuales tiene al menos un generador sincrónico. En $t=0 \mathrm{~s}$ se conecta una carga de 10kVA a la microrred 1, produciéndose una caída en la frecuencia. En $t=5 \mathrm{~s}$, se interconectan las dos microrredes produciéndose una mejora en la desviación de frecuencia.En el tercer caso, en dos microrredes idénticas, el inversor de $10 \mathrm{~kW}$ es reemplazado por SG idénticos. La Figura 14 muestra la variación de frecuencia para dos microrredes que contienen sendos generadores sincrónicos. En $t=0 \mathrm{~s}$ se conecta una carga de $10 \mathrm{kVA}$ a la microrred 1, produciéndose una caída en la frecuencia. En $t=7 \mathrm{~s}$, se interconectan las dos microrredes produciéndose una mejora en la desviación de frecuencia. Es notable la disminución del sobrepico en la frecuencia al conectar las dos microrredes.

\section{Conclusiones}

El control por pendiente garantiza la repartición de potencia en una microrred que incluye generadores sincrónicos. Al interconectar microrredes, las desviaciones respecto a la frecuencia de referencia son mejoradas aumentando la capacidad del sistema para atender variaciones bruscas de carga. Esta mejora se debe al aumento de la capacidad del sistema en conjunto, y en el caso que se encuentre conectados a las microredes generadores sincronicos, la inercia es mayor comparada 
con la generacion renovable utilizando inversores. Mediante los resultados simulados y pesentados en este articulo se puede apreciar una de las ventajas de la coordinacion y control cooperativo de multiples microredes. Nótese que la inteconexión entre microrredes puede disminuir la frecuencia de una de ellas, pero mejorarla en otra, mejorándola en todo el conjunto, a la vez que se incrementa la capacidad del sistema.

De esta manera, los resultados de simulación presentados demuestran las mejoras de la regulación de frecuencia utilizando el aumento de inercia de microrredes Interconectadas.

\section{REFERENCIAS}

[1] H. Farhangi, "The path of the smart grid," IEEE Power and Energy Magazine, vol. 8, no. 1, pp. 18-28, 2010.

[2] R. H. Lasseter, "MicroGrids," 2002 IEEE Power Engineering Society Winter Meeting. Conference Proceedings, 2002, pp. 305-308 vol.1.

[3] R. H. Lasseter, "Smart distribution: Coupled microgrids," Proceedings of the IEEE, vol. 99, no. 6, pp. 1074-1082, 2011.

[4] S. Rivera, A. M. Farid and K. Youcef-Toumi, "A multi-agent system transient stability platform for resilient self-healing operation of multiple microgrids," ISGT 2014, Washington, DC, 2014, pp. 1-5.

[5] Rivera, S., Farid, A. M., \& Youcef-Toumi, K, “A multi-agent system coordination approach for resilient self-healing operation of multiple microgrids," Industrial Agents: Emerging Applications of Software Agents in Industry, 2014, pp. 1-20.

[6] A. R. Bergen, Power systems analysis. Pearson Education India, 2009.

[7] E. Mojica-Nava, N. Quijano, and A. Pavas, "Dynamic population games for hierarchical microgrid management," in 2013 4th IEEE/PES Innovative Smart Grid Technologies Europe, ISGT Europe 2013, 2013.

[8] J. Rocabert, A. Luna, F. Blaabjerg, and I. Paper, "Control of Power Converters in AC Microgrids," IEEE Transactions on Power Electronics, vol. 27, no. 11, pp. 4734-4749, 2012.

[9] J. M. Guerrero, J. C. Vasquez, M. Savaghebi, J. D. Hoz, and H. Martín, "Hierarchical Control of Power Plants with Microgrid Operation," Annual Conference on
IEEE Industrial Electronics Society, pp. 3006-3011, 2010 .

[10] F.A. Zúñiga-Cortés, E.F Caicedo-Bravo y D.M López-Santiago. "Gestión óptima de la potencia eléctrica en una microrred conectada, basada en el algoritmo genético para optimización multiobjetivo MOGA", UIS Ingenierias, vol. 15, no. 2, pp. 17-33, jul-dic 2016,doi: 10.18273/revuin.v15n2-2016002.

[11] E.E Gaona Garcia, T Morales Vega. C.L Trujillo Rodríguez, F Santa María. "Esquemas de trasmisión de datos en una Microrred a través de una Infraestructura de medición avanzada", UIS Ingenierías, vol. 15, no. 2, pp. 85-92, jul-dic 2016, doi:10.18273/revuin.v15n22016007.

[12] A. Tuladhar, H. Jin, T. Unger, and K. Mauch, "Parallel operation of single phase inverter modules with no control interconnections," Proceedings of APEC 97 Applied Power Electronics Conference, vol. 1, pp. 94100, 1997.

[13] E. Coelho, P. Cortizo, and P. Garcia, "Small signal stability for single phase inverter connected to stiff AC system," Conference Record of the 1999 IEEE Industry Applications Conference. Thirty-Forth IAS Annual Meeting, vol. 4, pp. 2180-2187, 1999.

[14] J. M. Guerrero, N. Berbel, J. Matas, L. G. De Vicuna, and J. Miret, "Decentralized control for parallel operation of distributed generation inverters in microgrids using resistive output impedance," IECON Proceedings, Industrial Electronics Conference, vol. 54, no. 2, pp. 5149- 5154, 2006.

[15] T. L. Vandoorn, B. Meersman, J. D. M. De Kooning, and L. Vandevelde, "Directly-coupled synchronous generators with converter behavior in islanded microgrids," IEEE Transactions on Power Systems, vol. 27, no. 3, pp. 1395-1406, 2012.

[16] F. Dörfler, J. W. Simpson-porco, and F. Bullo, "Breaking the Hierarchy : Distributed Control and Economic Optimality in Microgrids," vol. 3, no. 3, pp. 241-253, 2016.

[17] J.D. Bastidas Rodríguez, C.A Ramos Paja, "Types of inverters and topologies for microgrid applications" UIS Ingenierías, vol. 16, no. 1, pp. 7-14, ene-jun 2017. 